\title{
LOS BLOQUES ARADORES DE "LAGUNA HELADA" (URBIÓN): UN EJEMPLO SINGULAR DE SOLIFLUXIÓN ACTIVA EN LA CORDILLERA IBÉRICA
}

\author{
ALIPIO GARCÍA DE CELIS - Pablo ARROYO PÉREZ - Alberto GANDÍA \\ FERNÁNDEZ \\ Departamento de Geografía, Universidad de Valladolid
}

\begin{abstract}
RESUMEN: En este trabajo se analizan los bloques aradores localizados en una ladera conformada a la pared de un antiguo circo glaciar, en un medio de alta montaña mediterránea (los Picos de Urbión, Cordillera Ibérica). El clima en estas montañas es mediterráneo pero en condiciones de gran altitud: es decir, con temperaturas bajas y escasez estival de precipitaciones. A pesar de unas condiciones de tipo mediterráneo los bloques aradores manifiestan claras evidencias de estar activos, y su movimiento parece estar relacionado con procesos de solifluxión o gelifluxión. En el entorno de los bloques aradores aparecen otras formas debidas a la solifluxión en laderas que también parecen activas, como lóbulos y terrazas, aunque estas formas no son objeto de estudio en este trabajo.

Se han tomado las medidas de 55 bloques aradores, y se han equipado diez de ellos con varillas para confirmar y medir el movimiento actual. Se ha analizado el suelo situado bajo los bloques y se ha medido la temperatura del suelo alrededor y bajo varios de ellos. Estamos ante una forma de gran singularidad por su aparente actividad y de gran significado ambiental, por su fragilidad y por ser buenos indicadores de la existencia o no de condiciones de alta montaña, lo que le dota de gran interés científico.
\end{abstract}

PALABRAS CLAVE: Picos de Urbión, bloques aradores, alta montaña mediterránea, solifluxión.

ABSTRACT: In this work we analyze the ploughing blocks located in different situations, all of these around old glacial cirques, in an alpine-mediterranean high mountain environment (the peaks of Urbion, Iberian Range, Soria province, Castilla-Leon, Spain). The climate in these mountains is mediterranean but in high altitude conditions: cold and dry. In spite of these conditions, ploughing blocks show clear evidence of activity, movement linked, we believe, to solifluction or gelifluction processes. Beside the ploughing blocks there are other solifluction features that seen active, such as solifluction lobes and banks. These features are not analyzed in this work.

We have measured around 50 ploughing blocks. The measured parameters are these: First, the slopes (tilt and highs): at a range between 2000 and 2100 meters high, and $5^{\circ}-30^{\circ}$ tilt. Second, the measures of each block, that show a wide range of size: from cobble to big boulders, all of them of a very cohesive quartz-sandstone. Third, the measures of grooves and front mounds. Ploughing blocks are located on slopes faced both north and south, but the evidences of recent movements are very different in each case. Around ten ploughing blocks have been equipped to probe and measure the probable movement. The high rarity and environmental significance of these features such as high mountain conditions indicators, near the limits of the periglacial environment, is the reason of their high scientific value and their character as a very important geomorphological heritage.

KEY WORDS: ploughing blocks, solifluction, mediterranean high mountain.

$\boldsymbol{R} \boldsymbol{E} \boldsymbol{S} \boldsymbol{U} \boldsymbol{M} \boldsymbol{E}:$ Nous étudions les blocs labourant localisés dans la parois d'an antique cirque glacial (Pics d'Urbión, Chaîne Ibérique, Province de Soria, Castilla y León, Espagne), dans un 
milieu d'haute montagne alpine-méditerranéen. Le climat de ces montagnes c'est méditerranéen (été fortement sèche, automne, hiver et printemps humide), mais en conditions de grand altitude (températures toujours froids). Les blocs labourant montrent évidences d'activité, un mouvement en rapport, nous croyons, avec processus actives de solifluxion or gélifluxion. Au coté des blocs labourant ils sont d'autres formes de solifluxion aussi actives, tels que lobes et bancs. Ces formes ne sont pas analysées dans ce travail. Nous avons mesuré 50 blocs labourant. Les paramètres mesurés sont les suivantes: Première, la versant (côte et altitude) : les blocs ce sont situées parmi les 2000 et les 2100 mètres, et en une versant avec $5-30^{\circ}$ côte. Second, les mesures de chaque bloc, lesquelles montrent un gros range de taille : depuis pierres même grandes blocs, touts il d'arenisque cuartzitique très cohésive. Troisième, les mesures des sillons et des accumulations frontales de chaque bloc. Les blocs sont localisés dans une versant orienté au nord. Dix blocs ont eu monitorisés pour traiter d'éprouver et mesurer son mouvement. Nous sommes devant unes formes très rares et d'une signifiante environnemental très important, puisque ce sont bon indicateurs de conditions d'haute montagne, prochaines a les milieux périglaciaires. C'est par là, ce sont formes d'une grande importance scientifique et de grande valeur patrimoniale.

MOTS-CLÉS: blocs labourant, solifluxion, haute montagne méditerranée.

RESUMO: Neste trabalho analizam-se os blocos de deslizamento localizados numa ladeira ao lado da parede dum antigo circo glaciar de uma alta montanha mediterrânea (Picos de Urbión, Cordilheira Ibérica). O clima é de carácter mediterrâneo de altitude, isto é, com temperaturas baixas e escassez de chuvas estivais. Apesar das condições de tipo mediterrâneo os blocos de deslizamento apresentam evidências de estarem activos e o seu movimento parece relacionar-se com processos de solifluxão e gelifluxão. Ao redor dos blocos de deslizamento aparecem outras formas causadas por processos de deslizamento em vertentes activas, como lóbulos e terraços, ainda que estas formas não sejam objecto deste estudo.

Usámos 55 blocos de deslizamento, dez deles dotados com varinhas para confirmar e medir o deslocamento actual. Analisou-se a camada de subsolo dos blocos medindo-se a temperatura do solo em seu redor e debaixo de algumas das suas partes. Apresentam uma forma muito singular pela atividade aparente e de grande significado ambiental, pela sua fragilidade e por serem bons indicadores da existência ou não de condições de alta montanha, o que lhes dá um grande interesse científico.

PALAVRAS CHAVE: Picos de Urbión, blocos de deslizamento, alta montanha mediterrânea, solifluxão.

\section{INTRODUCCIÓN.}

Los bloques aradores son una forma de relieve propia de los movimientos en masa lentos en medios periglaciares e infraperiglaciares (creep de helada, gelifluxión, solifluxión), consistente en fragmentos de roca de tamaño bloque que aparecen individualizados en una ladera, en la cual se mueven hacia abajo más deprisa que el regolito circundante, quedando como resultado de este desplazamiento una acumulación de material delante del bloque y una depresión o surco detrás (TUFNELL, 1972, 237-238. WASHBURN, 1979, 223). El término bloque arador implica un proceso -un tipo específico de movimiento de ladera lento- y unas formas -el propio bloque, el material acumulado y el surco- (ALLISON AND DAVIES, 1996, 202). Este tipo de forma ha sido encontrada por nosotros en las partes más altas de la Sierra de Urbión (Cordillera Ibérica), y con signos claros 
de actividad actual. En este trabajo presentamos los primeros resultados de la investigación: contexto geomorfológico y geoecológico en el que están insertos, características morfométricas y monitorización para tratar de comprobar y medir su movimiento. Nuestra intención es comprobar que se trata de un proceso activo y en funcionamiento, medir su ritmo y velocidad y buscar una explicación de la aparente actividad de unos procesos poco frecuentes en las montañas de la Península Ibérica. La importancia de esta investigación radica, en primer lugar, en que la demostración de la existencia de un piso infra-periglaciar activo (CHARDON, 1986, 217) permitiría definir la existencia de medios de alta montaña en la Cordillera Ibérica, de acuerdo con la definición clásica (TROLL, 1973, 146. PRICE, 1986, 5). En segundo lugar, es importante conocer la existencia, las características y el funcionamiento de medios periglaciares en las montañas ibéricas, pues se trata de medios extremadamente sensibles y frágiles, y por tanto buenos indicadores del cambio climático. Finalmente, es importante el conocimiento detallado de los medios fríos en estas montañas por su carácter aislado, frágil y singular, que les concede un alto valor científico y didáctico, así como un atractivo turístico que por otra parte puede significar una amenaza a la conservación de estas formas.

\section{II. ÁREA DE ESTUDIO}

El macizo montañoso de los Picos de Urbión alcanza los 2.200 metros de altitud (Urbión, $2.228 \mathrm{~m}$ ), y está situado en el interior de la Península Ibérica, en plena Cordillera Ibérica, alejado tanto del mar Mediterráneo (300 km en línea recta) como del océano Atlántico $(150 \mathrm{~km})$, mares de los que está separado no sólo por la distancia, sino también por la disposición de otros relieves montañosos (MAPA 1)

El clima es de tipo mediterráneo -lluvias en invierno, otoño y primavera, mientras que el verano es muy seco-, pero la altitud introduce un matiz de claro enfriamiento: periodo de heladas muy amplio (ningún mes libre de heladas), gran amplitud térmica tanto diaria como anual y estaciones muy contrastadas. No existen datos termopluviométricos de la montaña, pues los observatorios más cercanos son los situados en el pueblo de Vinuesa y en el puerto de montaña de Santa Inés (CUADRO 1).

A partir de los datos aportados por estos observatorios, es posible suponer que la isoterma anual de $0^{\circ}$ pueda situarse entre los 2.000 y los 2.100 metros de altitud (ARNÁEZ Y GARCÍA RUIZ, 2000, 119), lo cual, teóricamente, permitiría plantear la existencia de condiciones periglaciares a partir de esa altitud. Es significativo que a esta altitud aparece también el límite superior de la vegetación arbórea: por encima de los 2.000 metros hay muy pocos árboles, ninguno supera los dos metros de altura, y la mayoría presentan formas degradadas por la 
acción del viento y el hielo (FOTO 1). La vegetación dominante pasa a ser matorrales de brezo (Erica $s p$.) y praderas de Festuca $s p$. mezcladas con afloramientos o depósitos de rocas desnudas.

Mapa 1. Localización de los Picos de Urbión

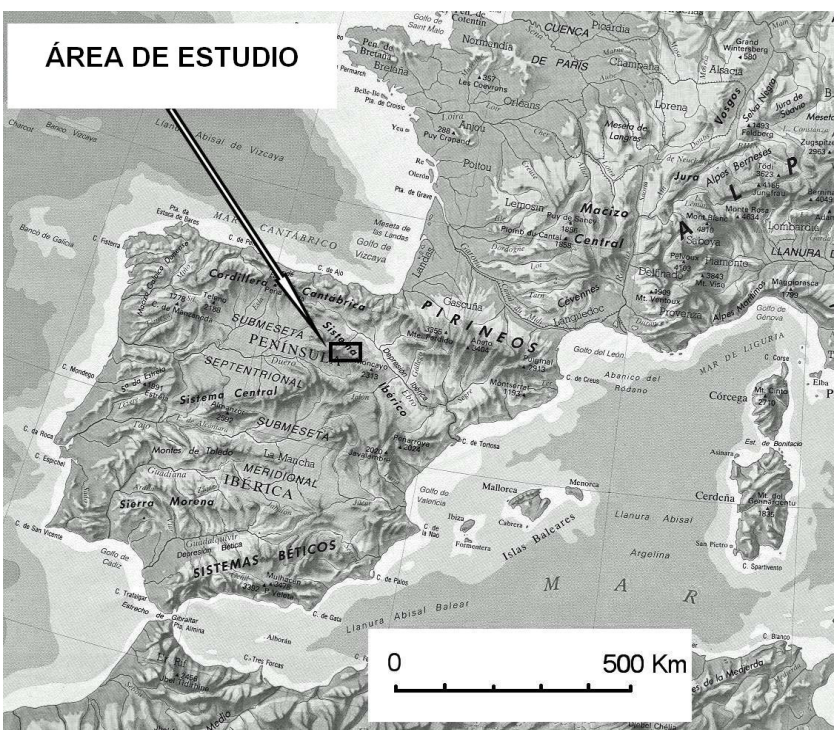

Foto 1. Aparte de los datos climáticos y de la presencia de los fenómenos geomorfológicos que aquí se estudian, la existencia de unas condiciones de alta montaña parecen manifestarse también en el hecho de que a partir de los $1.950 \mathrm{~m}$. de altitud desaparece el bosque, y la vegetación pasa a estar formada por árboles pequeños (menos de dos metros de altura) y deformados por el viento y las heladas, junto a un matorral de ericáceas (Erica sp.) y praderas de Festuca sp.

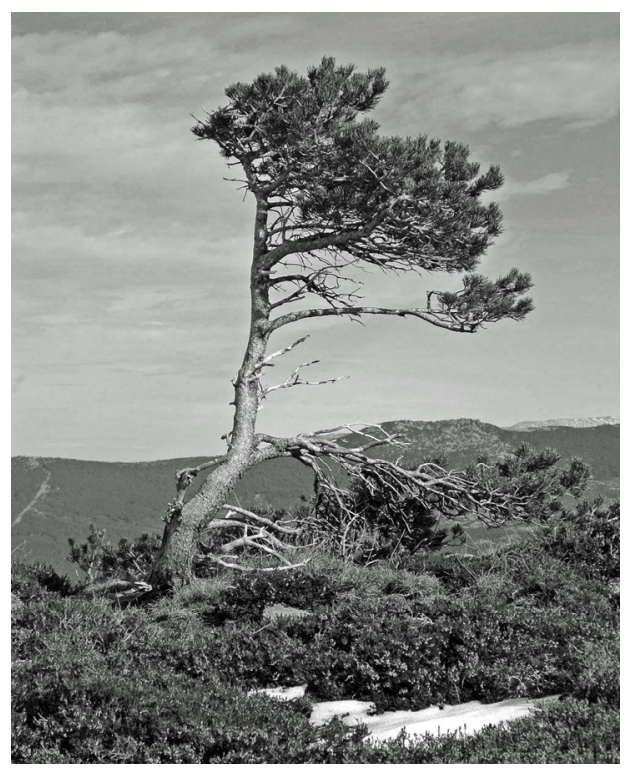


Cuadro 1. Observatorios termopluviométricos más cercanos

\begin{tabular}{lcc}
\hline Nombre del observatorio: & Vinuesa & Puerto de Santa Inés \\
Altitud $(\mathrm{m})$ : & 1.107 & 1.326 \\
Núm. de años con datos: & 38 & 11 \\
Temperatura media anual $\left({ }^{\circ} \mathrm{C}\right):$ & 9,6 & 7,6 \\
Precipitación media anual $(\mathrm{mm}):$ & $1.001,5$ & $1.141,4$ \\
\hline
\end{tabular}

FUENTE: FORTEZA (1981)

\section{CONTEXTO GEOMORFOLÓGICO DE LOS BLOQUES ARADORES: EL CIRCO DE "LAGUNA HELADA".}

El conjunto principal de bloques aradores, y el que presenta síntomas más evidentes de actividad, se localiza junto a la "Laguna Helada". Esta laguna se asienta en el fondo de un gran recuenco correspondiente a un antiguo circo glaciar (MAPA 2). En efecto, durante la última glaciación se formaron en los Picos de Urbión glaciares que en la fase de máximo llegaron a prolongarse en lenguas de varios kilómetros de longitud que descendieron por los valles hacia la parte baja de la montaña (GANDÍA, 2005; Sanz, 2001; THORNES, 1968).

Sobre la pared del antiguo circo glaciar de "Laguna Helada" aparece hoy un recubrimiento formado por un regolito de fragmentos heterométricos de arenisca envueltos en una matriz fina (arenosa) y muy fina (limoarenosa). La ladera presenta dos partes diferenciadas: una superior, con pendiente de más de $20^{\circ}$ y con mayor abundancia de clastos (regolito clasto-soportado) con numerosos bloques de grandes dimensiones (más de 1 metro de eje mayor). Otra parte inferior, con pendiente inferior a $10^{\circ}$, regolito matriz-soportado y bloques de menores dimensiones. Los bloques aradores se localizan sobre este regolito, en ambos sectores de la ladera.

Superficialmente, el modelado de la ladera, además de los bloques aradores, incluye lóbulos y bancos de solifluxión con la misma frescura de formas y apariencia de activos que los bloques aradores. El resto de la ladera, sobre la antigua pared del circo, presenta un recubrimiento muy fino de regolito, aflorando la roca del sustrato en numerosos puntos. Aparecen algunos nichos de nivación hoy no funcionales, así como antiguas morrenas de nevero y morrenas correspondientes a las fases finales de la actividad glaciar. También son destacables algunos deslizamientos y desprendimientos recientes.

En la culminación de la ladera aparece un escarpe rocoso, correspondiente al borde del antiguo circo glaciar. Este escarpe rocoso está formado por el afloramiento de un estrato de areniscas cuarcíticas de grano grueso ("Grupo Urbión", Cretácico inferior, IGME, 1986, 19), que buza hacia el suroeste unos $20^{\circ}$. 
Mapa 2. Mapa geomorfológico detallado del circo de "Laguna Helada" (Picos de Urbión)

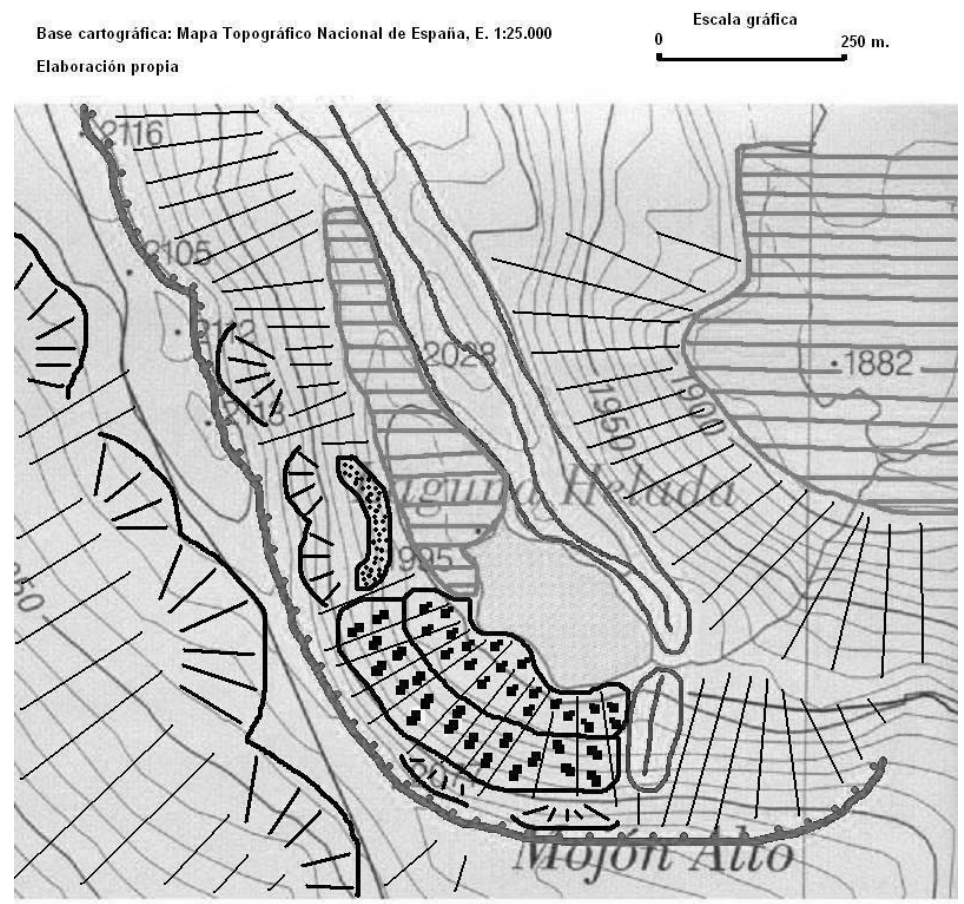

Formas de origen periglaciar y nival

Formas de origen glaciar

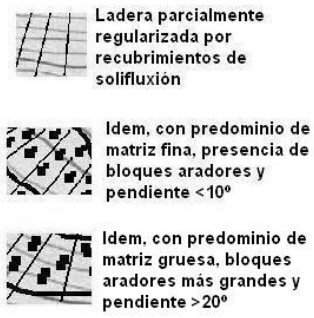

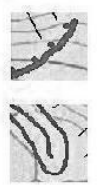

Borde de circo Nicho de nivación Morrena de nevero

Otras formas

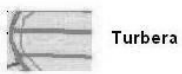

La parte inferior de la ladera enlaza con la superficie del lago llamado "Laguna Helada", que da nombre al conjunto. Este lago ocupa la cubeta de sobreexcavación del fondo del antiguo circo glaciar. El lago y el circo están delimitados por el umbral que cierra la cubeta de sobreexcavación, que corresponde a un segundo estrato de areniscas cuarcíticas de grano grueso, más resistentes a la 
abrasión glaciar que las areniscas cuarcíticas de grano fino sobre las que está formada la pared del circo glaciar. El umbral glaciar está sobredimensionado por la acumulación sobre él de un depósito morrénico correspondiente a una fase de estabilización final, en el proceso de deglaciación, cuando el glaciar quedó recluido al ámbito del circo.

\section{MEDIDAS DE LOS BLOQUES ARADORES.}

Se midieron 55 bloques, y el resumen de los datos puede observarse en el CUADRO 2.

Cuadro 2. Resumen de los principales parámetros de los 55 bloques aradores medidos

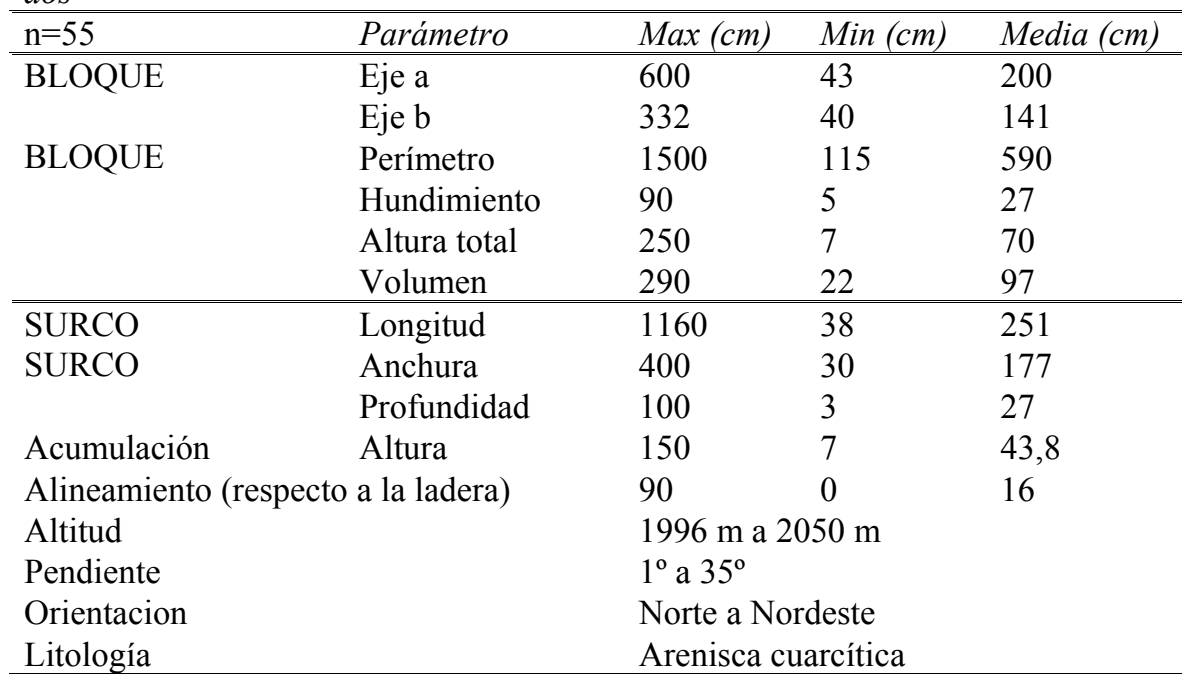

En conjunto, los bloques aradores se sitúan a una altitud entre 1995 y 2060 metros y ocupan una parte de la ladera orientada al Nornoreste. La litología de todos los bloques aradores de Urbión es arenisca cuarcítica. El tamaño de los bloques es bastante grande, con un eje mayor medio de 2 metros y un eje menor de 1,41 metros, siendo la rectangular y piramidal las formas dominantes de los bloques. Los bloques aradores se encuentran bastante hundidos en el suelo. En relación con esto, los surcos no son muy largos, con una longitud media de 2,51 metros y una anchura de 2,57, dominando en planta las formas rectas y en nicho con una sección parabólica y en cuna principalmente, estas depresiones están ocupadas por agua durante la primavera. La mayoría de los bloques aradores tienen una acumulación fronto-lateral bien desarrollada, con una altura media de $44 \mathrm{~cm}$, que a veces presenta grietas y partes con el suelo desnudo e irregularidades y desprendimientos en los frentes que pueden ser indicadores de activi- 
dad actual. Los bloques están muy alineados respecto a la orientación de la ladera con un $63 \%$ por debajo de $15^{\circ}$ de desviación.

Como hemos señalado, en función de la pendiente se distinguen dos tramos en la ladera: uno inferior, entre 1995 y 2015 m, de pendiente suave -en torno a $5^{\circ}$-; y otro superior, a una altitud entre 2015 y $2060 \mathrm{~m}$ con una pendiente mayor de $20^{\circ}$. En el sector inferior los bloques aradores están bastante individualizados, dando como resultado muy buenos ejemplos de bloques aradores con unas microformas asociadas bien definidas claramente identificables (FOTO 2).

Foto 2. Grupo de bloques aradores de la parte baja de la ladera. Puede observarse la menor pendiente, con respecto a la parte alta de la ladera que se observa al fondo, y el carácter más fino del regolito sobre el que se sitúan los bloques. Destaca el pliegue o acumulación de suelo situada en la parte frontal de los bloques, de grandes dimensiones y forma perfectamente nítida, lo que parece evidenciar un movimiento de empuje actual o muy reciente.

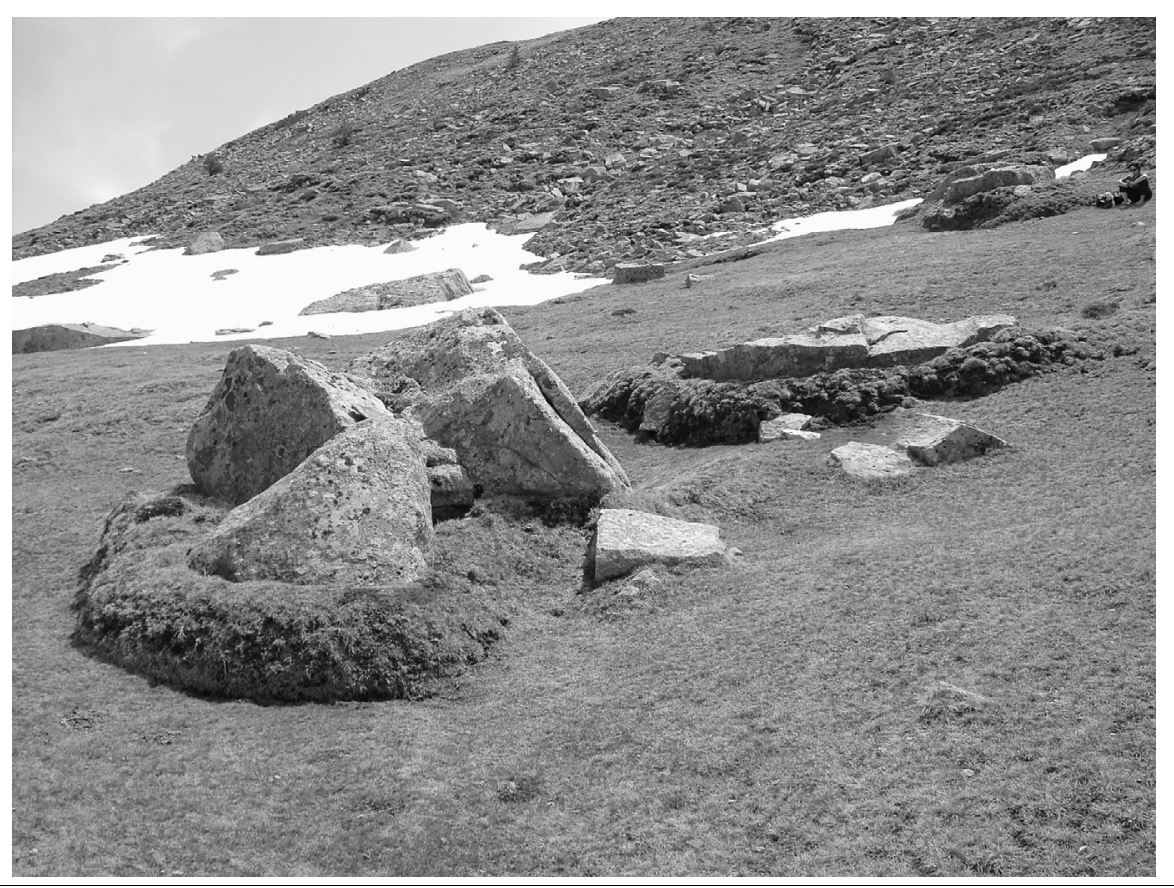

En el tramo superior la pendiente es más acusada y la disposición de los bloques aradores más caótica, están asentados sobre un manto de solifluxión grosero y aparentemente activo, y también se encuentran ejemplos notables de blo- 
ques aradores, algunos de grandes dimensiones (FOTO 3), y que, al igual que en la parte más baja, muestran claros síntomas de actividad actual.

Foto 3. Un gran bloque de la parte alta de la ladera. Puede observarse el carácter más grosero del regolito que tapiza la ladera, así como la mayor pendiente.

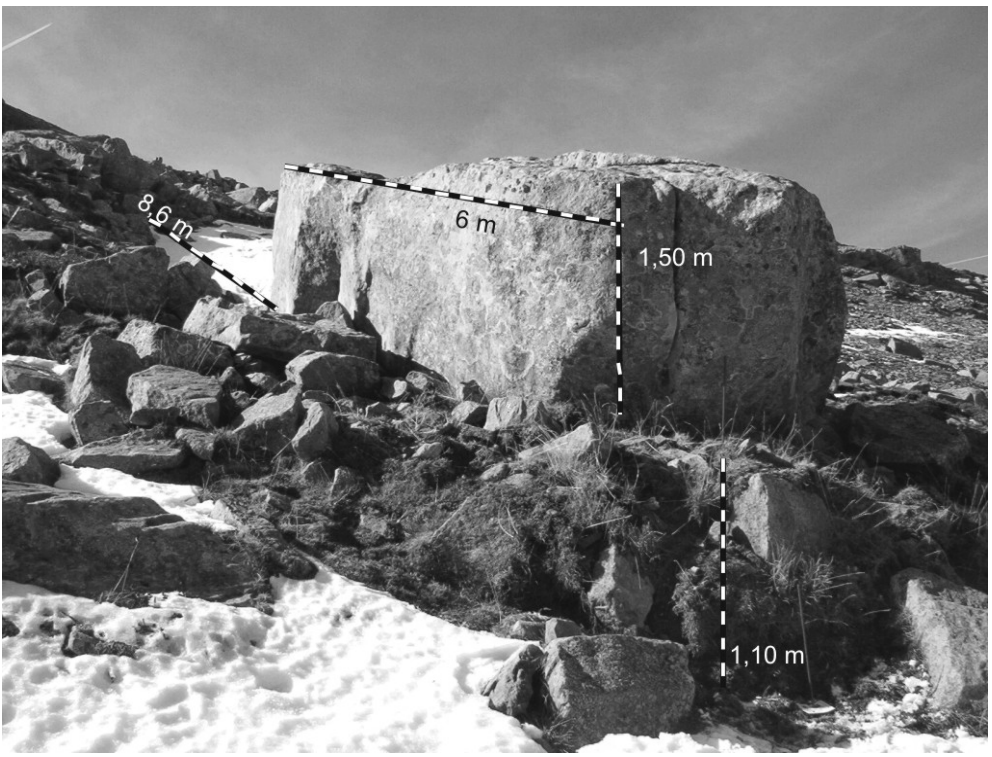

\section{CARACTERÍSTICAS DEL SUELO ALREDEDOR Y DEBAJO DE LOS BLOQUES ARADORES}

Las características del suelo, especialmente el porcentaje de finos y el contenido de materia orgánica, tienen influencia directa en la formación de bloques aradores (WILSON 1993: 24-25). En nuestro caso (FOTO 4) el suelo se caracteriza por su textura arcillo-limosa. Presenta un horizonte superficial de unos 20-25 $\mathrm{cm}$. de espesor en el que se concentran las raíces (Fото 5). Éstas son algo más abundantes y más gruesas bajo los bloques aradores, lo cual puede estar relacionado con la conductividad térmica de éstos. Bajo este horizonte superficial aparece un horizonte profundo, sin apenas raíces y con estructura moderada de tipo columnar grueso, con desarrollo de cutanes. El color de ambos horizontes oscila entre marrón ( $\left.7^{\prime} 5 \mathrm{YR} 4 / 3\right)$ y marrón rojizo (5YR 4/4). En primavera el suelo aparece empapado en agua a partir de una profundidad de $30-40 \mathrm{~cm}$.

Hemos constatado que la temperatura del suelo es sensiblemente más alta debajo del bloque que en su entorno inmediato en la época de deshielo primaveral, siendo esta diferencia térmica mayor según avanza el día (CUADRO 3), lo que 
confirma la importancia de las diferencias en la transmisión de calor por conductividad térmica entre el bloque y el suelo y su posible relación con el movimiento del bloque arador (BALLANTYNE, 2001, 281; REID Y NESJE, 1988, 31).

Foto 4. En uno de los bloques aradores situado en la parte baja de la ladera se excavó una pequeña cata para observar las características del suelo bajo el bloque, en el amontonamiento frontal y en el surco.

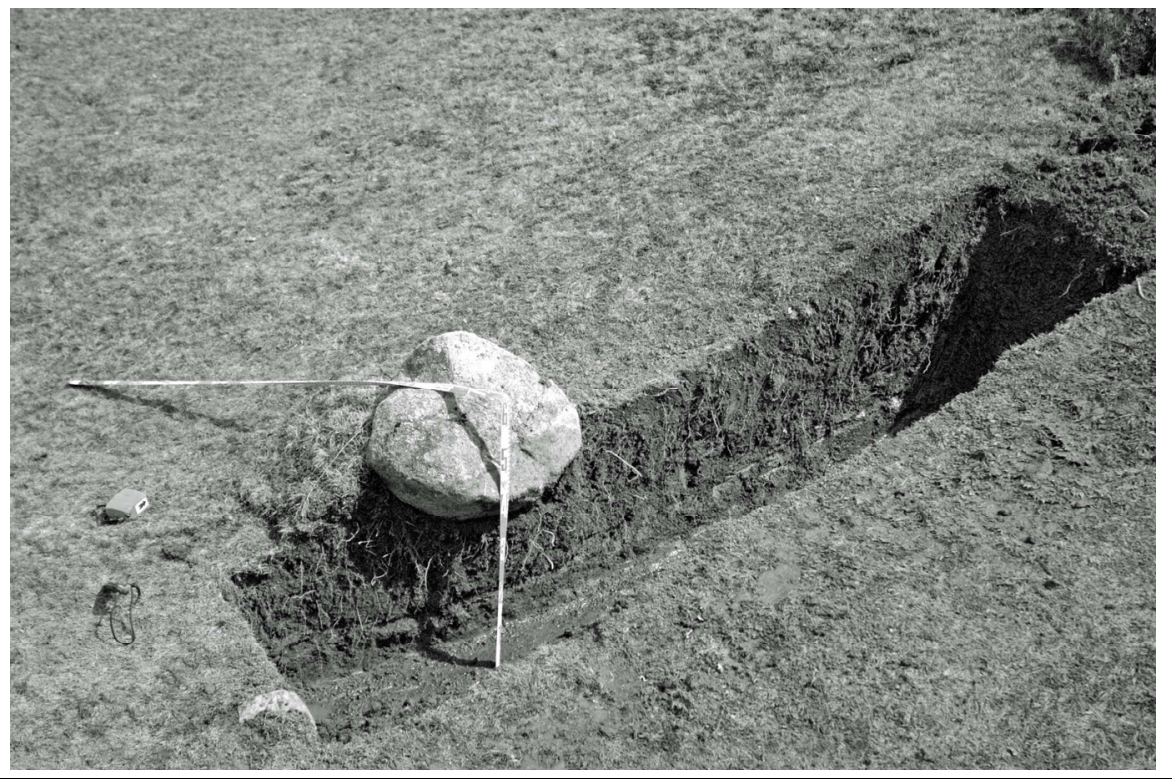

Cuadro 3: Medición de la temperatura del suelo en un día de primavera, tomada mediante termómetro de sonda (a $15 \mathrm{~cm}$. de profundidad) bajo el bloque y a veinte centímetros de éste, en diez bloques aradores.

\begin{tabular}{ccccccc}
\hline $\begin{array}{c}\text { Bloque } \\
n^{o}:\end{array}$ & \multicolumn{2}{c}{${ }^{\circ} \mathrm{C}$ 11:00 horas } & \multicolumn{2}{c}{$T^{\circ} \mathrm{C}$ 18:00 horas } & \multicolumn{2}{c}{ Variación } \\
\cline { 2 - 7 } & A $20 \mathrm{~cm}$. & Bajo el bloque & A $20 \mathrm{~cm}$. & Bajo el bloque & $11: 00$ & $18: 00$ \\
\hline 1 & 8,4 & 12,3 & 4,9 & 7,2 & 2,3 & 3,9 \\
2 & 8,7 & 13,3 & 5,9 & 7,1 & 1,2 & 4,6 \\
3 & 6,1 & 10,1 & 6,5 & 6,9 & 0,4 & 4 \\
4 & 7,3 & 10,5 & 6,5 & 7,8 & 1,3 & 3,2 \\
5 & 8,8 & 6,3 & 8,7 & 5,9 & $-2,8$ & $-2,5$ \\
8 & 9,6 & 11,3 & 7,6 & 8,5 & 0,9 & 1,7 \\
9 & 8,8 & 17,7 & 8,8 & 11,8 & 3 & 8,9 \\
10 & 9,6 & 11,2 & 6,7 & 9,4 & 2,7 & 1,6 \\
\hline
\end{tabular}


Foto 5. Detalle del horizonte superficial del suelo situado bajo el bloque, donde puede verse la concentración de raices y el encharcamiento del horizonte subyacente.

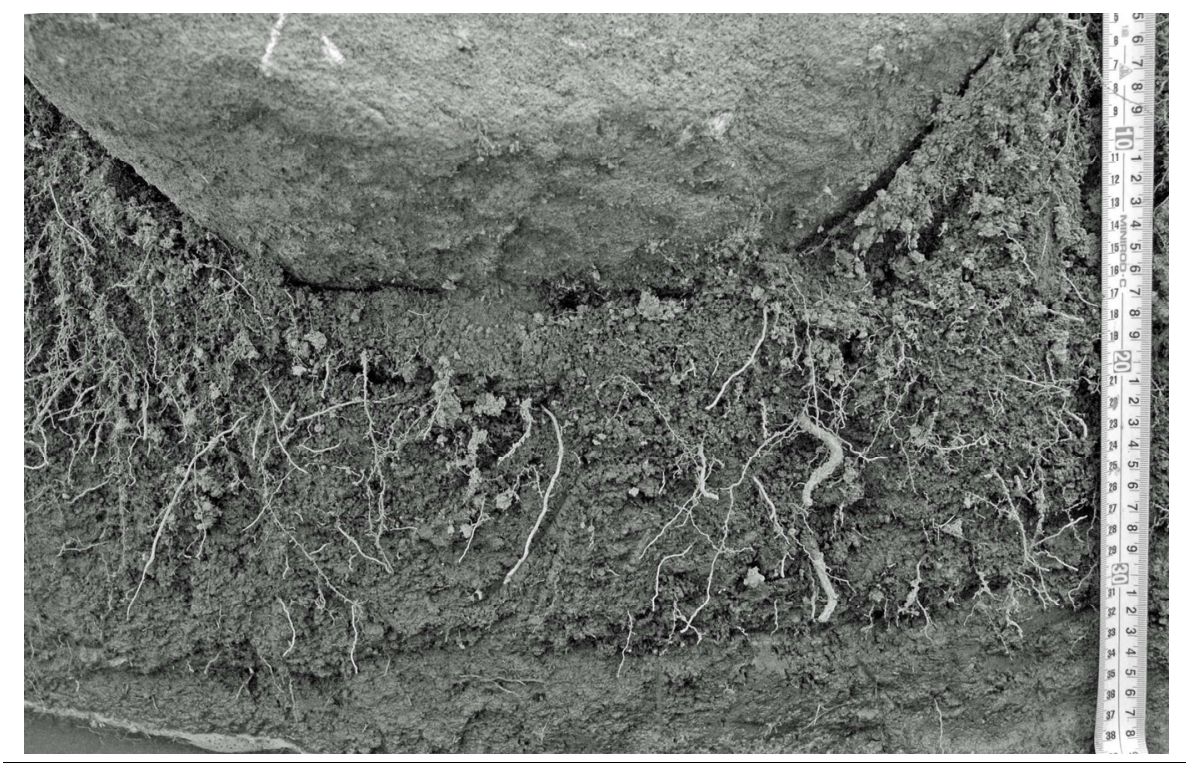

\section{MEDICIÓN DE LA VELOCIDAD DEL MOVIMIENTO DE LOS BLOQUES ARADORES}

Se equiparon 10 bloques aradores (FOTO 6) con el objetivo de obtener datos que nos permitan confirmar y medir la actividad actual de los bloques. Se instalaron varillas metálicas de $50 \mathrm{~cm}$. de longitud, clavadas en el suelo a una profundidad variable, de entre 20 y $30 \mathrm{~cm}$. Las varillas se clavaron verticales y en la parte delantera del bloque, una sobre la acumulación frontal, situada lo más próxima posible al bloque, y otra varilla delante de la acumulación frontal. Se tomaron las medidas resultantes desde el extremo superior de cada varilla hasta un punto marcado en el bloque, y se midió también la parte sobresaliente de cada varilla.

Las varillas se instalaron en el mes de noviembre, y las medidas se repasaron en mayo, tras el deshielo primaveral. Se ha observado que el comportamiento de las varillas es diferente en función de su cercanía al bloque: las varillas que se clavaron más próximas a los bloques se han movido más que las que se clavaron por delante de la acumulación frontal, que apenas se han movido. Las más cercanas a los bloques han sido empujadas hacia afuera una media de 3 centímetros. También ha variado la distancia con respecto al bloque: las varillas más cercanas al bloque se han inclinado ladera abajo, perdiendo su verticalidad, 
y ha aumentado su distancia respecto al bloque una media de 2-3 centímetros. Las variaciones son claramente medibles en los bloques situados en la parte alta de la ladera. En la parte baja de la ladera aparentemente no ha habido variaciones en las medidas, o éstas han sido poco significativas.

Foto 6. Diez de los bloques aradores han sido equipados con dos varillas cada uno, con la intención de verificar y medir su movimiento. Las varillas fueron clavadas verticalmente en el suelo a una profundidad de al menos $20 \mathrm{~cm}$, una inmediatamente delante del bloque, a unos pocos centímetros, y otra situada delante del amontonamiento frontal.

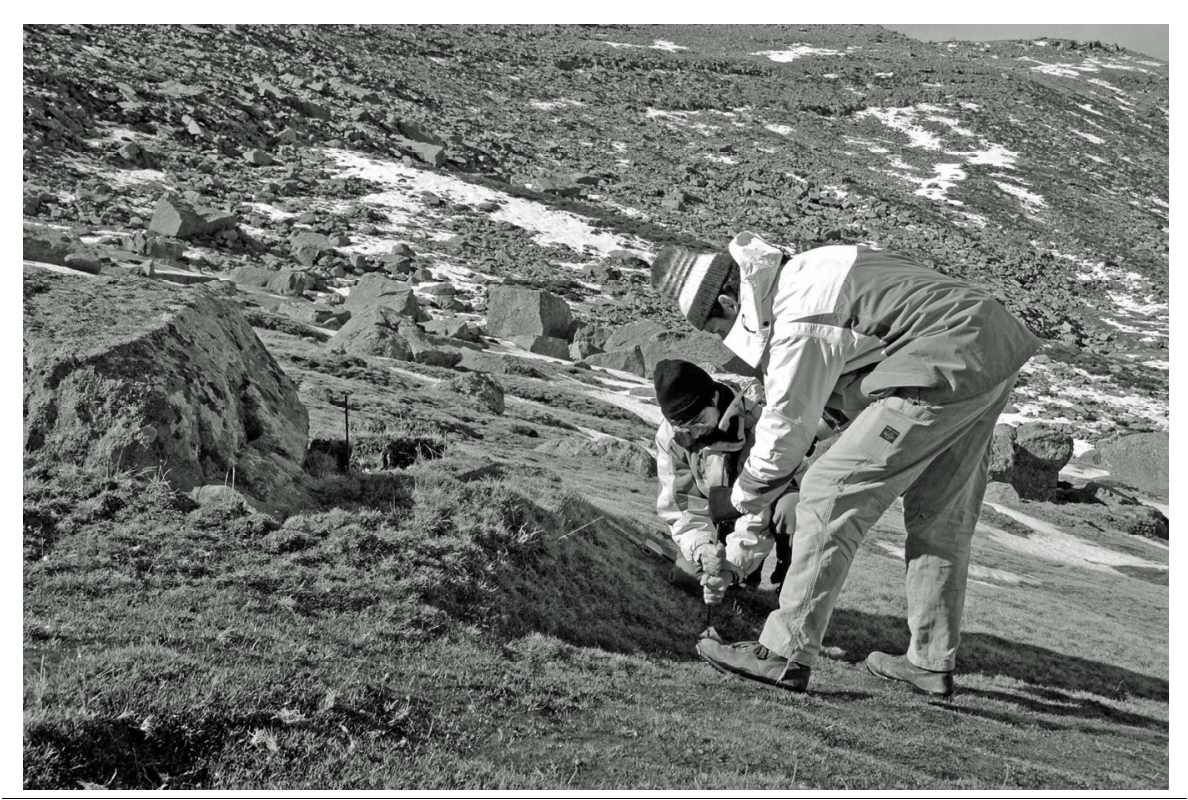

Es evidente que entre los meses de noviembre y mayo (invierno e inicio de la primavera) los bloques de la parte alta de la ladera se han movido, pero las varillas también se han movido, probablemente por la acción de empuje del hielo del suelo. Al estar clavadas delante de los bloques, resulta difícil estimar cuál ha sido el movimiento del bloque con respecto a la ladera. Pensamos que la monitorización se puede mejorar clavando otras varillas a ambos lados del bloque, alejadas de él y de la acumulación frontal, para tratar de medir la velocidad y el ritmo del desplazamiento de los bloques respecto del regolito sobre el que están asentados. 


\section{DISCUSIÓN Y CONCLUSIONES: LOS BLOQUES ARADORES COMO INDICADORES DE CONDICIONES DE ALTA MONTAÑA EN URBIÓN}

Los bloques aradores constituyen un elemento geomorfológico que permite observar la dinámica de los medios de alta montaña, dado que esta forma periglaciar es relativamente frecuente y un buen indicador para valorar el significado actual de los procesos activos de tipo infra-periglaciar en las montañas ibéricas.

El relieve de los sectores más altos de la Sierra de Urbión, por encima de los 1900 metros, está marcado por las formas de excavación y acumulación glaciar y un importante retoque periglaciar. La parcela de trabajo se localiza en uno de los circos glaciares más importantes, el de "Laguna Helada", donde las formas de solifluxión asociadas a un regolito abundante en fracción fina, son muy frecuentes. En la pared del circo son frecuentes los grandes bloques de arenisca y en algunos sectores se aprecia una interesante dinámica de ladera actual, con la presencia de algunos movimientos de ladera recientes (deslizamientos, desprendimientos). Aparecen bloques aradores por encima de los 1.995 metros de altitud. El número de bloques aradores existentes es lo suficientemente elevado como para considerarlo una forma de modelado significativa. El estudio de los surcos y las acumulaciones frontales, así como los primeros datos de los bloques monitorizados, permiten afirmar que los bloques aradores de Urbión están activos, son funcionales, si bien la técnica empleada no permite avanzar datos fiables que permitan cuantificar el movimiento.

Por otra parte, la presencia de bloques activos coincide, en altitud, con los ejemplares individuales arbóreos más extremos del límite del bosque. La escasa talla de los árboles, sus deformaciones y su presencia dispersa marcan lo que puede considerarse como el límite superior de la vegetación arbórea, la timberline.

Se cumplirían así todos los criterios para poder hablar de la existencia de un medio de alta montaña, definido por la presencia de un piso alpino o periglaciar activo, probablemente en su fase menos avanzada, es decir, un piso infraperiglaciar, en estas montañas del interior de la Península Ibérica.

A partir de esta constatación, la intención de los autores es continuar midiendo el ritmo y velocidad de la actividad de los bloques aradores, para avanzar en el conocimiento del grado de actividad de este medio infra-periglaciar, y disponer de datos para tratar de evaluar, en un futuro cercano, posibles alteraciones relacionadas con procesos de cambio climático a nivel global que, en estas 
montañas mediterráneas, pudieran significar la desaparición de unos medios tan frágiles como son los de la alta montaña.

\section{BIBLIOGRAFÍA}

ALLISON, R.J. AND DAVIES, K.C. (1996): «Ploughing blocks as evidence of down-slope sediment transport in the English Lake District». Zeitschrift für Geomorphologie, 106, 199-219.

ARNAEZ, J. Y GARCIA RUIZ, J.M. (2000): «El periglaciarismo en el Sistema Ibérico Noroccidental». En: PEÑA, J.L., SANCHEZ-FABRE, M. y LoZANO, M.V. (eds.) Procesos y formas periglaciares en la montaña mediterránea. Teruel, Instituto de Estudios Turolenses, 113-126.

BALlantYNE, C.K. (2001): «Measurement and theory of ploughing boulder movement». Permafrost and Periglacial Prosesses, 12, 267-288.

CHARDON, M. (1984): «Montagne et haute montagne alpine. Criteres et limites morphologiques remarquables en haute montagne». Revue de Geographie Alpine, LXXII, 23, 213-224.

FORTEZA, M (1982): Caracterización agroclimática de la provincia de Soria. Madrid. Ministerio de Agricultura, Pesca y Alimentación.

GANDIA, A. (2005) Geomorfología del Alto Urbión. Memoria de Investigación de Doctorado. Valladolid, Universidad de Valladolid. Inédito.

IGME (1986): Mapa Geológico de España E.1:50.000. Quintanar de la Sierra. Madrid, Instituto Geológico y Minero de España.

PRICE, L.W. (1986): Mountains and man. A study of process and environment. Berkeley, University of California.

REID, J.R. Y NESJE, A. (1988): «A giant ploughing block, Finse, southern Norway». Geografiska Annaler. 70A, 27-33.

SANZ, E. (2001): Las montañas de Urbión, Cebollera y Cabrejas: Geomorfología y patrimonio geológico. Soria, Diputación de Soria, Colección Temas Sorianos.

THORNES, J.B. (1968): «Glacial and periglacial features in the Urbion Mountains, Spain». Estudios Geológicos, XXIV, 249-255

TROLL, C. (1973): «La Geoecología y la diferenciación a escala planetaria de los ecosistemas de alta montaña». Geographica, 2, 143-155.

TUFNELL, L. (1972): «Ploughing blocks with special reference to north west of England». Biuletyn Periglacjanly, 21, 237-270.

WASHBURN, A. L. (1979): Geocryology. A survey of periglacial process and environments. London, Edward Arnold.

WILSON, P. (1993): «Ploughing-boulder characteristics and associated soil properties in the Lake District and southern Scotland». Scottish Geographical Magazine, 109, 1826. 